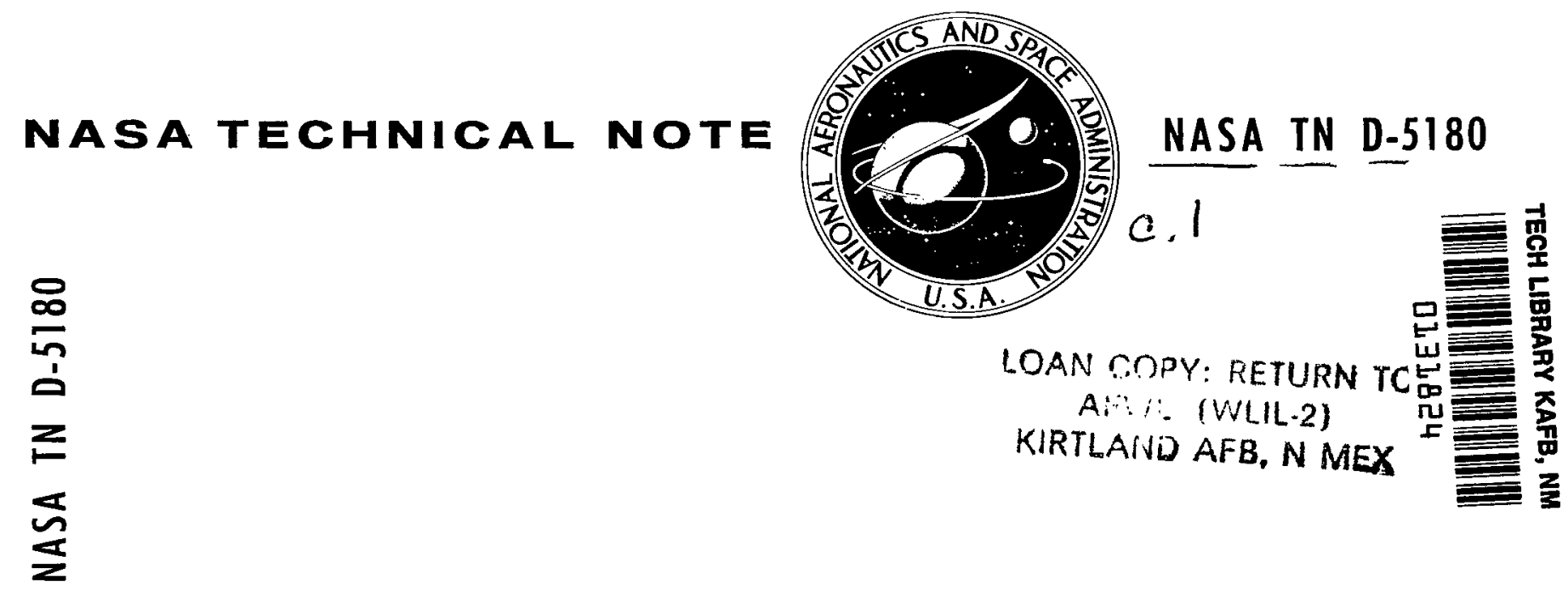

\title{
FIRST-ORDER SOLUTIONS FOR
} MUTUAL COUPLING BETWEEN WAVEGUIDES WHICH PROPAGATE TWO ORTHOGONAL MODES

by R. J. Mailloux

Electronics Research Center

Cambridge, Mass. 
FIRST-ORDER SOLUTIONS FOR MUTUAL COUPLING BETWEEN WAVEGUIDES WHICH PROPAGATE TWO ORTHOGONAL MODES

By R. J. Mailloux

Electronics Research Center

Cambridge, Mass.

\section{NATIONAL AERONAUTICS AND SPACE ADMINISTRATION}

For sale by the Cleoringhouse for Federal Scientific and Technical Information Springfield, Virginia 22151 - CFSTI price $\$ 3.00$ 


\section{ABSTRACT}

This paper describes a theoretical and experimental study of closely spaced open-ended waveguides radiating through a conducting ground plane. The waveguides are large enough in some cases to allow two orthogonally polarized propagating modes in each waveguide.

A first-order analysis is presented which is based upon the method of moments and uses a single-mode approximation to the aperture field for each polarization. An improved firstorder analysis is also presented which uses a higher order mode solution for the self admittances. Close agreement with experiment is demonstrated. 
FIRST-ORDER SOLUTIONS FOR MUTUAL COUPLING BETWEEN

WAVEGUIDES WHICH PROPAGATE TWO ORTHOGONAL MODES

By R. J. Mailloux

Electronics Research Center

\section{SUMMARY}

This report describes a theoretical and experimental study of closely spaced open-ended waveguides radiating through a conducting ground plane. The waveguides are large enough in some cases to allow two orthogonally polarized propagating modes in each waveguide.

A first-order analysis is presented which is based upon the method of moments and uses a single-mode approximation to the aperture field for each polarization. An improved first-order analysis is also presented which uses a higher order mode solution for the self admittances. Close agreement with experiment is demonstrated.

\section{INT RODUCT ION}

The realization of large-scale microwave phased arrays has focussed new interest on the problems of mutual coupling between radiating apertures. A previous survey of developments in this field has been included in an earlier paper (refs. 1 and 2).

There are two distinct approaches to phased array problems. Work on infinite arrays of waveguides has progressed rapidly to a point at which several authors (refs. 3, 4, 5, and 6) have used higher order mode representations of the aperture field and have obtained solutions that are capable of explaining certain anomalous behavior (resulting in deep nulls in the radiation pattern as the array is scanned). Farrell and Kuhn (refs. 3 and 4) point out that this behavior cannot be predicted using a single-mode solution. The second approach to the array problem assumes the point of view that each terminal pair leading to an antenna element is a single port and is coupled to every other port in the array by means of the mutual admittances or the array scattering matrix. Since the controlling integral equations are linear, this approach can be made completely rigorous if the mutual coupling terms or scattering coefficients are evaluated in the presence of the whole array, and if the computation includes all of the higher order modes. A solution for the coupling between two collinear coupled slots has recently been published which does account for a large number of modes (ref. 1). There is strong justification, however, to seek simpler yet accurate 
mutual coupling formulas for aperture antennas, since this method can be used directly for small arrays or for edge effects in large arrays; can be readily compared with experiment, and can be used to study infinite arrays directly or after transformation using the Poisson summation formula (ref. 7). An example of the use of this type of analysis to study the performance of large scanned phased arrays is included in reference 8 .

The number of modes required to attain a specified accuracy or to describe a particular phenomenon is strongly dependent on the formulation of the problem. This statement is best supported by the well-known variational formulation results (refs. 9 and 10) for the rectangular waveguide. These one-mode results are far more accurate than the one-mode solution to the integrated form of Lewin's basic integro-differential equation (ref. 2).

Another interesting point raised by the work of Farrell and Kuhn, and a particularly relevant one to the development of realistic mutual coupling formulas, is the existence of crosspolarized modes at the array face. These fields are always present for arrays of waveguides with finite walls, but if the waveguides can support two propagating orthogonally polarized modes, then large cross-polarized signals are reflected back into each waveguide at certain directions of scan even though the array was driven with only one polarization. In a related development, Amitay et al. (ref. 11) have recently developed a match optimization procedure for finite phased arrays which requires a knowledge of the inter-element coupling coefficients for both polarizations. The authors compared their theoretical work with an experiment, and used measured values of the coupling coefficients to perform the optimization.

This paper presents a study of mutual coupling between two waveguides which radiate through a common ground plane. The waveguide walls are parallel to one another, but the relative waveguide position and orientation are arbitrary. Both components of polarization are considered in the coupling formulas, and the end result is a solution for the scattering matrix of the four-port network. The results are compared with experiments and with those of a more accurate theory in the special case of collinear slot coupling.

\section{FIRST-ORDER SOLUTIONS \\ INCLUDING TWO ORTHOGONAL POLARIZATIONS}

In this simplified formulation each of two orthogonal polarizations is represented by a single mode within each waveguide. The waveguide orientation is shown in Figure 1. The fields in free space are represented by the free-space dyadic Green's 


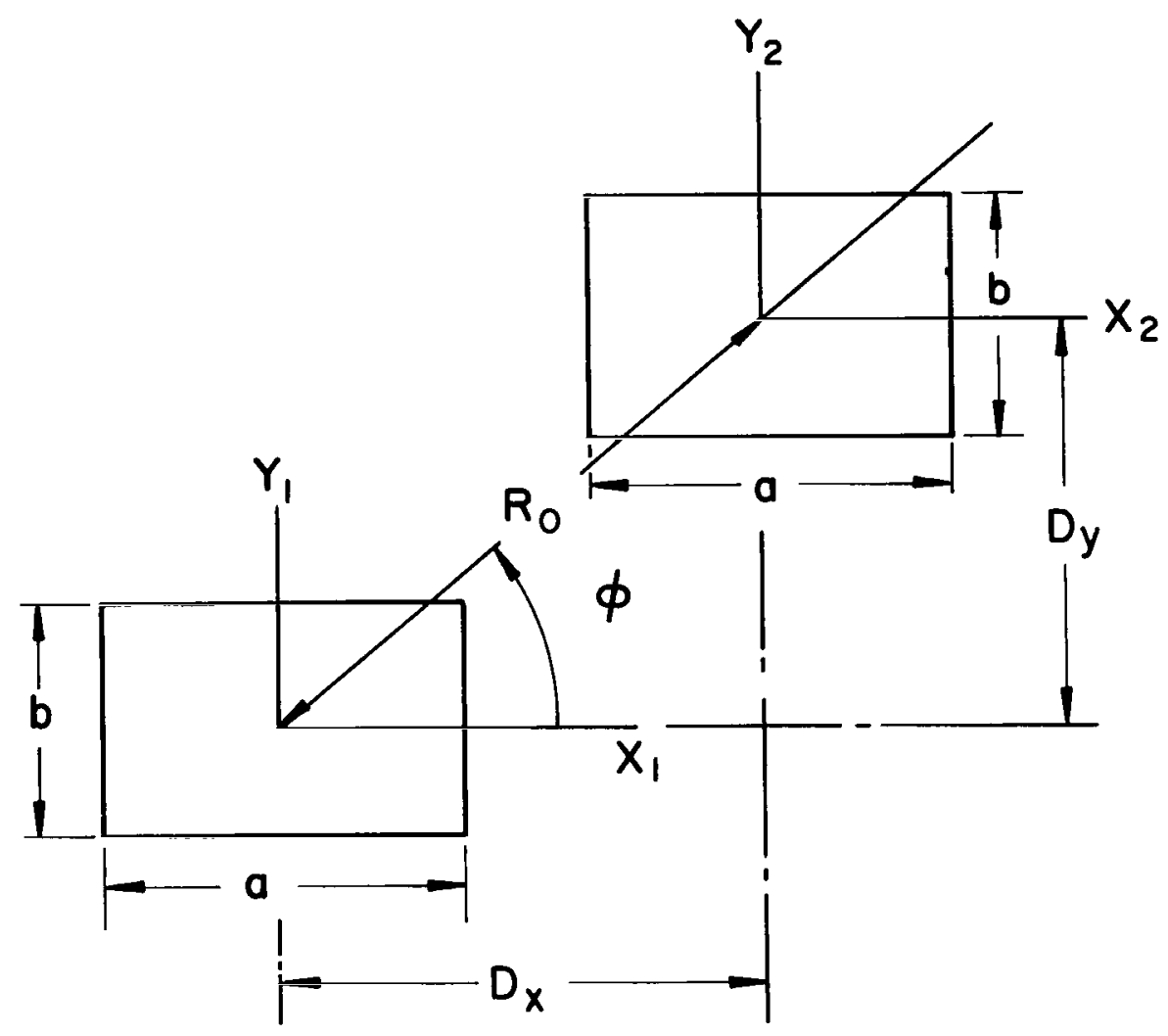

Figure 1.- Coupled waveguide geometry

function, and the fields in each waveguide are represented by the transverse field (ref. 12) expansion. Details are given in Appendix I. Equating the tangential magnetic fields in the apertures gives the resulting set of integral equations (ref. 7, p. 100; ref. 13, p. 177). At waveguide No. 1:

$$
\begin{aligned}
& \left.\hat{z} \times I_{1}(0) \bar{h}_{1}\left(x_{1}, y_{1}\right)+I_{2}(0) \bar{h}_{2}\left(x_{1}, y_{1}\right)\right] \\
& =\hat{z} \times \sum_{p=1,2} \int_{S_{p}} \int_{\underline{y}}\left(\bar{r}_{1}, \bar{r}_{p}^{\prime}\right) \cdot\left[\hat{z} \times E\left(x_{p}^{\prime}, y_{p}^{\prime}\right)\right] d S_{p}^{\prime}
\end{aligned}
$$


At waveguide No. 2 :

$$
\begin{aligned}
\hat{z} \times\left[I_{3}(0) \bar{h}_{3}\left(x_{2}, y_{2}\right)+I_{4}(0) \bar{h}_{4}\left(x_{2}, y_{2}\right)\right] \\
\quad=\hat{z} \times \sum_{p=1,2} \int_{S_{p}} \int_{y}\left(\bar{r}_{2}, \bar{r}_{p}^{\prime}\right) \cdot\left[\hat{z} \times E\left(x_{p}^{\prime}, y_{p}^{\prime}\right)\right] d S_{p}^{\prime}
\end{aligned}
$$

where

$$
\underline{\mathrm{y}}\left(\bar{r}_{\mathrm{k}}, \bar{r}_{\mathrm{p}}^{\prime}\right)=\underline{\mathrm{y}}\left(\left|\bar{r}_{\mathrm{k}}-\bar{r}_{\mathrm{p}}^{\prime}\right|\right)=\underline{y}\left(r_{\mathrm{kp}}\right)
$$

and

$$
\begin{aligned}
& r_{11}=\sqrt{\left(x-x_{1}^{\prime}\right)^{2}+\left(y-y_{i}^{\prime}\right)^{2}} \\
& r_{12}=\sqrt{\left(x_{2}+D_{x}-x_{1}^{\prime}\right)^{2}+}\left(y_{2}+D_{y}-y_{1}^{\prime}\right)^{2} \\
& r_{21}=\sqrt{\left(x_{2}^{\prime}+D_{x}-x_{1}\right)^{2}}+\left(y_{2}^{\prime}+D_{y}-y_{1}\right)^{2} \\
& \left.r_{22}=\sqrt{\left(x_{2}-x_{2}^{\prime}\right)^{2}+\left(y_{2}\right.}-y_{2}^{\prime}\right)^{2}
\end{aligned}
$$

In these equations, the field subscripts 1 and 2 refer to the normal $\left(\mathrm{H}_{10}\right)$ and orthogonal $\left(\mathrm{H}_{01}\right)$ polarizations in waveguide No. 1, and the subscripts 3 and 4 refer to the normal and orthogonal polarizations in waveguide No. 2. The position subscripts $\mathrm{x}_{\mathrm{p}}$ and $\mathrm{Yp}$ are indicated in Figure 1 . The integrals over $\mathrm{S}_{\mathrm{p}}$ are
therefore integrals over apertures 1 and 2 .

There are a number of ways of proceeding to a solution commencing with Eqs. (1) and (2). Levis (ref. 10) multiplied these equations in scalar fashion by the aperture electric field and was able to show that the variational form which he obtained led to admittance parameters which are stationary with respect to the variations in the assumed fields. Lyon et al., (ref. 13) scalarly multiplied these equations by $\left(\hat{z} \times h_{i}\right)$ for each $\bar{h}_{i}$ present on the left side of Eqs. (1) and (2). The resulting equations are exact, but the solution was carried out by assuming that only 
one mode was present in the waveguide aperture and that the waveguide-backed ground-plane slot had a total magnetic field equal to the incident magnetic field (as is the case for scattering by a hole in an infinite screen). It can be shown that if the solution were carried out rigorously, the value $I_{2} / V_{1}$ computed using this second assumption with the second waveguide terminated in a matched load will be $\left(1+\mathrm{y}_{33} / \mathrm{y}_{0}\right) / 2$ times the correct value. The analysis presented here begins with the procedure outlined by Iyon et al., but includes the additional orthogonally polarized component. Moreover, instead of separating the equations using the magnetic field assumption the four equations are written directly in terms of their admittance matrix, and the scattering coefficients are obtained by performing a matrix inversion and using the well-known relations between the admittance matrix and the scattering matrix of an $N$-port network (ref. 12, p. 13). In accordance with this outline, Eq. (1) is scalarly multiplied consecutively by $z \times \bar{h}_{1}$ and $z \times \bar{h}_{2}$, and Eq. (2) by $\mathrm{z} \times \overline{\mathrm{h}}_{3}$ and $\mathrm{z} \times \overline{\mathrm{h}}_{4}$. The resulting equations are integrated over their respective apertures and reduced by orthogonality to the four equations below. This procedure is an example of the method of moments (ref. 14).

$$
\begin{aligned}
& \left\{\begin{array}{l}
I_{1} \\
I_{2}
\end{array}\right\}=\sum_{p=1,2} \int_{S_{1}} \mathrm{ss}_{1} \int_{S_{p}} \mathrm{ds}_{\mathrm{p}}^{\prime}\left\{\begin{array}{l}
\overline{\mathrm{h}}_{1}\left(\mathrm{x}_{1}, \mathrm{y}_{1}\right) \\
\overline{\mathrm{h}}_{2}\left(\mathrm{x}_{1}, \mathrm{y}_{1}\right)
\end{array}\right\} \cdot \underline{\mathrm{y}}\left(\overline{\mathrm{r}}_{1}, \overline{\mathrm{r}}_{\mathrm{p}}^{\prime}\right) \cdot\left[\mathrm{z} \times \mathrm{E}\left(\mathrm{x}_{\mathrm{p}}^{\prime}, \mathrm{y}_{\mathrm{p}}^{\prime}\right)\right] \\
& \left\{\begin{array}{l}
I_{3} \\
I_{4}
\end{array}\right\}=\sum_{p=1,2} \int_{S_{2}} \mathrm{ds} \int_{S_{p}} \mathrm{ds}_{p}^{\prime}\left\{\begin{array}{l}
{\overline{h_{3}}}_{\mathrm{p}_{2}}\left(\mathrm{x}_{2}, \mathrm{y}_{2}\right) \\
\overline{\mathrm{h}}_{4}\left(\mathrm{x}_{2}, \mathrm{y}_{2}\right)
\end{array}\right\} \cdot \underline{\underline{y}}\left(\bar{x}_{2}, \bar{r}_{\mathrm{p}}^{\prime}\right) \cdot\left[\mathrm{z} \times \mathrm{E}\left(\mathrm{x}_{\mathrm{p}}^{\prime}, \mathrm{y}_{\mathrm{p}}^{\prime}\right)\right]
\end{aligned}
$$

After expanding the admittance dyad, writina the fields in terms of the modal functions, and performing the numerical operations, these equations may be written using the admittance matrix notation

$$
\mathrm{I}=\mathrm{yV}
$$


where the admittance matrix coefficient satisfies reciprocity $\mathrm{Y}_{\mathrm{mn}}=\mathrm{Y}_{\mathrm{nm}}$ and symmetry relations $\mathrm{Y}_{11}=\mathrm{Y}_{33}, \mathrm{Y}_{22}=\mathrm{Y}_{44}, \mathrm{Y}_{32}=\mathrm{Y}_{14}$ ' $\mathrm{y}_{34}=\mathrm{y}_{12}$. With these relations, only six parameters need be defined to determine all the others.

$$
\begin{aligned}
& y_{11}=y_{1} c_{1} \Psi_{A}(0, a ; 0, b) \\
& y_{12}=y_{1} c_{1} \Psi_{B}(0, a ; 0, b) \\
& y_{13}=y_{1} c_{1} \Psi_{A}\left(D_{x}, a ; D_{Y}, b\right) \\
& y_{14}=y_{1} c_{1} \Psi_{B}\left(D_{x}, a ; D_{y}, b\right) \\
& y_{22}=Y_{2} c_{2} \Psi_{A}(0, b ; 0, a) \\
& y_{24}=y_{2} c_{2} \Psi_{A}\left(D_{y}, b ; D_{x}, a\right)
\end{aligned}
$$

The parameters $\Psi_{A}, \Psi_{B}, Y_{1}, Y_{2}, c_{1}$, and $c_{2}$ are defined in Appendix II.

The admittance parameters computed in this manner are not stationary with respect to the aperture field, although the self admittances are identical to those derived by Lewin (ref. 9) and, therefore, do represent the first-order stationary solution for an isolated waveguide. Though this self admittance is known to be accurate for most waveguide radiators, the slot conductance it predicts for the square waveguides considered later in this paper is up to 10 percent less than is predicted by the more accurate twelve-mode solution (ref. 1). The error in power coupling which this introduces is discussed in the following section.

Having thus defined all of the admittances, the scattering parameters may be obtained by matrix inversion using the equation

$$
S=(I-y)(I+y)^{-I}
$$

where

$$
\mathrm{y}_{\mathrm{mn}}=\mathrm{y}_{\mathrm{mn}} / \mathrm{y}_{\mathrm{m}}
$$

These scattering parameters will be the items of interest for the remainder of this paper. 
Before progressing to a discussion of the data, it is appropriate to mention several conclusions evident from a consideration of the symmetry of $\Psi_{A}$ and $\Psi_{B}$. The function $\Psi_{B}=0$ whenever $\mathrm{D}_{\mathrm{y}}$ or $\mathrm{D}_{\mathrm{x}}$ or both are 0 . Therefore, $\mathrm{y}_{12}$ is identically zero, and Y14 (and, hence, $S_{12}$ and $S_{14}$ ) is zero when the slots are arranged in either collinear or parallel fashion. In addition, it can also be shown that $\Psi_{\mathrm{B}}\left(\mathrm{D}_{\mathrm{X}}, \mathrm{a} ; \mathrm{D}_{\mathrm{y}}, \mathrm{b}\right)=\Psi_{\mathrm{B}}\left(\mathrm{D}_{\mathrm{Y}}, \mathrm{b} ; \mathrm{D}_{\mathrm{X}}, \mathrm{a}\right)$. This symmetry was used earlier to require that $\mathrm{y}_{14}=\mathrm{y}_{32}$, and $\mathrm{y}_{12}=\mathrm{y}_{34}=0$, but it also requires that if $a=b$, the coefficients $S_{12}$ and $S_{14}$ have even symmetry in $\phi$ about the angle $\phi=45$ degrees. In addition, all parameters have even symmetry in $\phi$ about $\phi:=0$, $\phi=90$ degrees and, hence, about $\phi=180$ degrees. For this reason, if $a=b$, only the parameters $s_{11}, S_{12}, s_{13}$, and $s_{14}$ need be computed in the restricted range $0 \leq{ }^{1} \phi \leq 90$ degrees to completely specify all other coupling parameters at all orientations.

\section{THEORETICAL AND EXPERIMENTAL RESULTS}

An experiment was conducted to compare with theoretical $r$ sults. All data were taken with the slots mounted on a 6-ft by 6-ft ground plane with the equipment located behind the ground plane. Absorbing material was used to minimize the reflections from the walls of the enclosing room and, in some cases (Figures $3 A$ and 4 ), the frequency was swept and the data averaged while making power-coupling measurements in order to minimize the error due to reflections. These measurements were repeatable to within about $0.5 \mathrm{~dB}$.

Since commercial dual-mode couplers were used to measure both polarizations of coupling at the received waveguide, a technique was based upon a previously published article (ref. 15), and required that an electric field probe be mounted perpendicular to the ground plane in order to establish a known phase relation between the two slots when they are excited together. When $\phi \neq 0$, the probe is placed midway between the slots on the line joining their centers and the probe reading is null when the slots are excited and in phase. When $\phi=0$, the electric field component $\mathrm{E}$ on this center line is zero because of symmetry, and so the probe is placed along a perpendicular bisector of the center to center line but away from this zero-field position. For this configuration, the probe reading is null when the slots are excited with equal out-of-phase signals. Other details of the phase-bridge calibration are included in the reference.

The phase reference for the orthogonally polarized coupled signal was obtained by exciting that waveguide and setting the electrical path length equal to that of the waveguide receiving the transmitted polarization. The resulting \pm 180 -degree phase 
ambiguity was resolved to \pm 360 degrees by correlation with the theoretical results. Coupled phases could have been measured by this technique to an accuracy of about \pm 4 degrees, if all external reflections were eliminated, the actual phase error in the presence of reflections was about \pm 10 degrees.

Figure 2 shows the amplitude and phase of the measured coupling and compares this with results computed using both the first-order theory and a more accurate nine-mode solution (ref. 1). The two computed values of power coupling are nearly the same for the lower frequencies, but as the frequency is increased, the first-order solution predicts somewhat less power coupling than either that observed or computed using the higher order mode solution. This deviation was greatest for the closest spaced slots for which the higher order mode coupling curve deviated quite visibly from a straight line. This deviation has been shown (ref. 2) to be due to the increased amplitude of higher order modes in the aperture field. The measured phase of the coupled signal shown in Figure $2 \mathrm{~B}$ follows the nine-mode solution very closely. The first-order solution predicts the phase to an accuracy of about 10 degrees except for the slots which are very closely spaced.

Figures 3 and 4 show the variation of power coupling and phase with frequency for square waveguides with several different values of $\phi$. These data are compared with experimental results over only a limited frequency range (8.5-9.7 GHz) because of the band-pass range of the dual-mode couplers. The measured coupling was always within about $1.5 \mathrm{~dB}$ of the theoretical data and exhibited a nearly identical variation. In order to determine how much of this error was due to using the first-order self admittance, the theoretical results were recomputed using a higher order (12-mode) self-admittance. These results are shown by the dotted line in Figure 3. The coupling computed by this improved first-order theory agrees to within about 1 dB with the measured data, and this is nearly equal to the absolute accuracy of the measuring system. The phases of the coupled signals are shown in Figures $3 B$ and 4. Unfortunately, the phase of $S_{13}$ was so nearly constant between $\phi=45$ and 90 degrees, as was the phase of $\mathrm{S}_{14}$ for $\phi$ between 30 and 60 degrees, that these phase changes with the angle $\phi$ could not be detected experimentally. The theoretical curves reflect the same characteristics.

These comparisons show that this simple theoretical treatment can yield very good quantitative information about mutual coupling. Figure 5 shows the dependence of $\mathrm{S}_{12}, \mathrm{~S}_{13}$, and $\mathrm{S}_{14}$ with position angle $\phi$ for various slot separations with $\mathrm{a} / \lambda=\mathrm{b} / \lambda=0.6$. This curve was computed using the improved first-order solution. The coefficient $S_{11}$ undergoes little change as these parameters are varied, and so is not plotted. Its value 


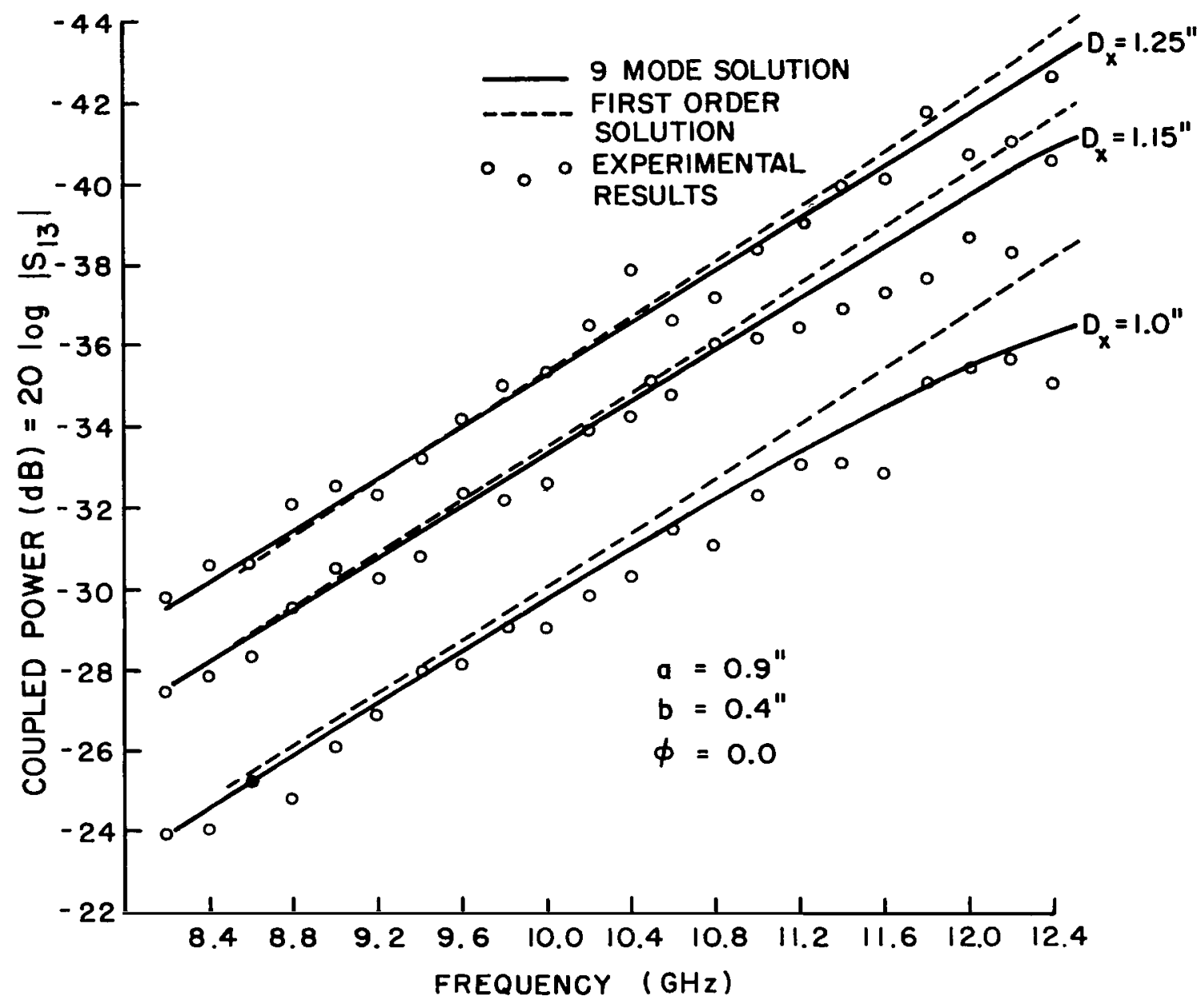

Figure 2A.- Comparison between two theories and experiment, collinear coupled waveguides, coupled power, theory vs experiment 


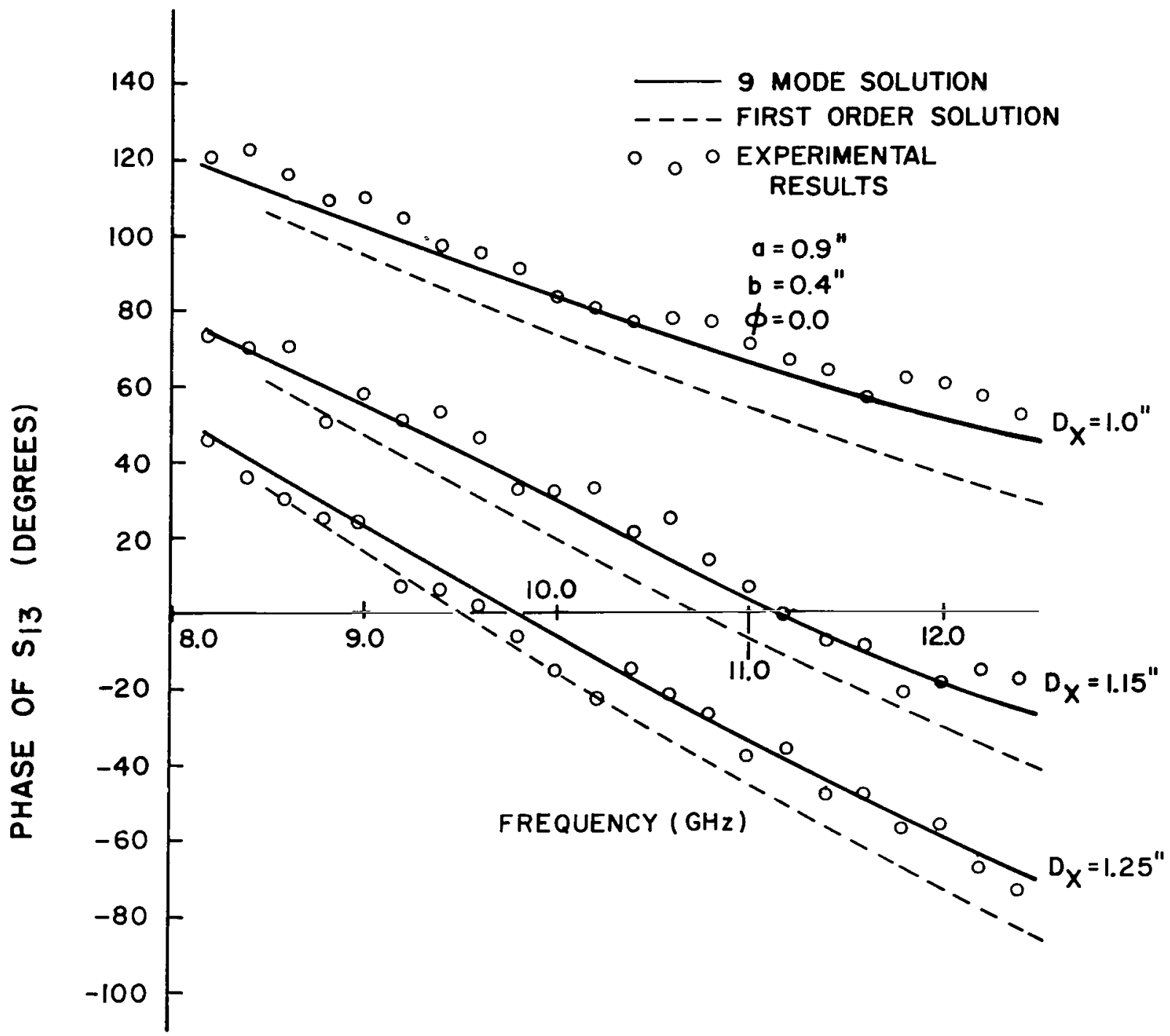

Figure 2B.- Comparison between two theories and experiment, collinear coupled waveguides, phase of $\mathrm{S}_{13}$, theory vs experiment 


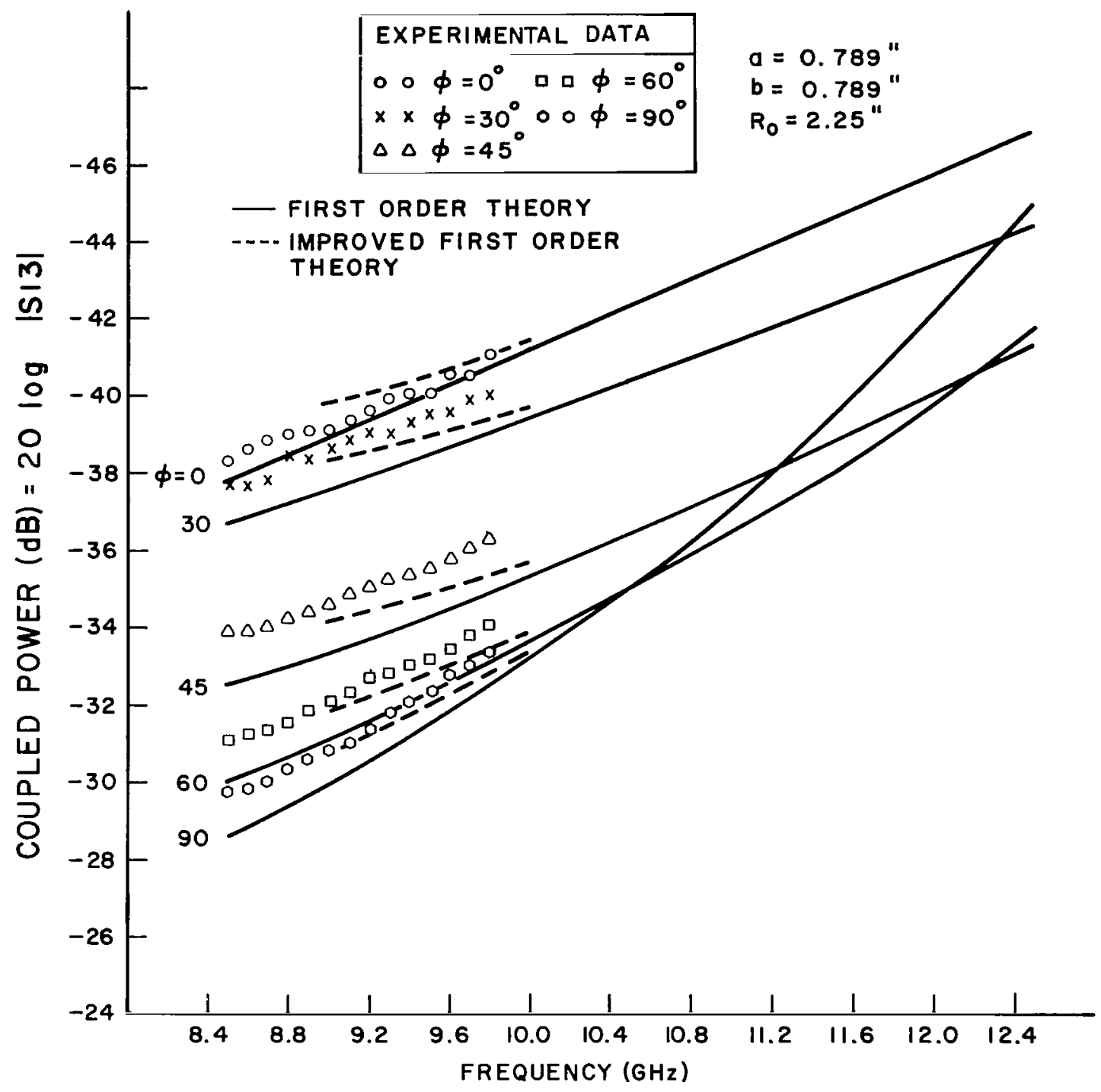

Figure 3A.- Comparison between first-order theory, improved first-order theory and experiment, coupled power, theory vs experiment 


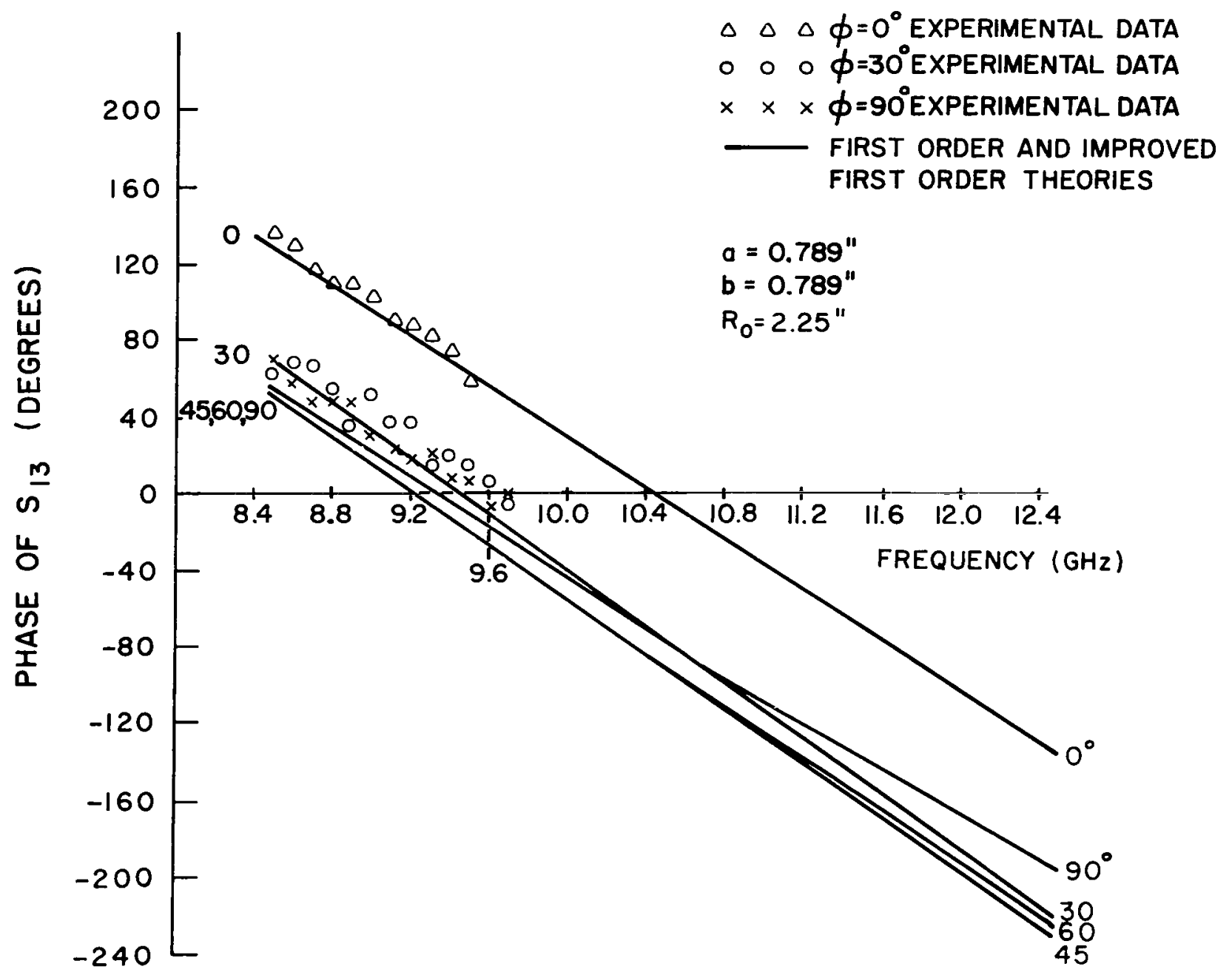

Figure 3B.- Comparison between first-order theory, improved first-order theory and experiment, phase of $S_{13}$, theory vs experiment 


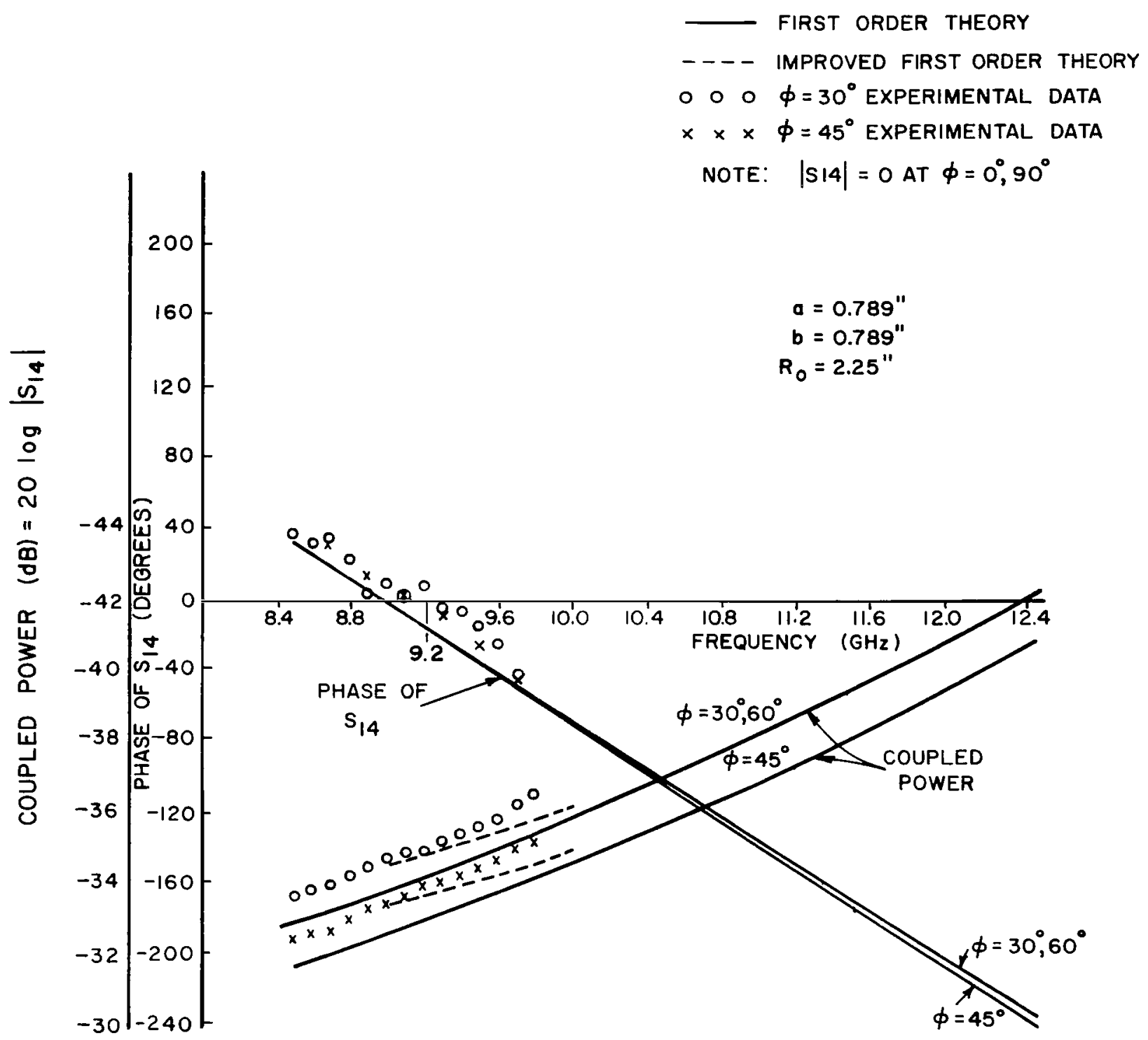

Figure 4.- Comparison between first-order theory, improved first-order theory and experiment, coupled power and phase of $\mathrm{S}_{14}$ 
is about $0.146-j 0.020 j$ As $R_{0} / \lambda$ is changed in Figure $5 \mathrm{~A}$, the coupling amplitude $\left|\mathrm{S}_{13}\right|$ at $\phi=0$ degree varies much faster than than at $\phi=90$ degrees. This is so because this amplitude varies asymptotically as $1 / R_{0}^{2}$ when $\phi=0$ and as $1 / R_{0}$ when $\phi=90$ degrees. Figure 5A shows, however, that for relatively close waveguide spacings the minimum coupling occurs at an angle between $\phi=0$ degree and $\phi=90$ degrees, and as the spacing is increased this minimum moves closer to its asymptotic limit $\phi=0$ degree. This coupling variation with $\phi$ is therefore a near field effect and so any attempt to describe the scattering parameter $s_{13}$ as the product of an angle variable and a space variable would fail to describe it completely.

The second important feature evident in Figure $5 \mathrm{~A}$ is that the amplitude of coupling into the orthogonal port $\left|\mathrm{S}_{14}\right|$ is larger than $\left|\mathrm{S}_{13}\right|$ for some angles of $\phi$. Therefore, this term must be considered when the waveguide geometry is such that this mode can propagate. The coefficient $\left|S_{12}\right|$ is also shown in this figure and behaves essentially like $\left|S_{14}\right|$ as $\phi$ is varied, though it is about an order of magnitude smaller. Both $\left|S_{12}\right|$ and $\left|S_{14}\right|$ are symmetric about $\phi=45$ degrees.

Figure $5 \mathrm{~B}$ shows variation in phase of $\mathrm{S}_{12}, \mathrm{~S}_{13}$, and $\mathrm{S}_{14}$ as the waveguide angle $\phi$ is varied. Of particular interest in these curves is the nearly constant phase change between curves of constant $R_{O} / \lambda$, indicating the expected phase delay with distance. The phase change in $S_{12}$ is about double that of $\mathrm{S}_{13}$ or $\mathrm{S}_{14}$ for the same change in $R_{0} / \lambda$, thus emphasizing that $S_{12}$ does not arise at the face of waveguide No. I itself but is due to the round trip coupling from waveguide No. 1 to waveguide No. 2 and then back again.

\section{CONCLUSION AND COMMENTS ABOUT APPROXIMATE SOLUTIONS}

This study has shown that the first-order or improved firstorder mutual coupling approximations can be used to provide an accurate and convenient formulation for the four-port scattering parameters of two arbitrarily located waveguides radiating through a ground plane. This model has also shown that the cross-polarized component of the coupled signal is of the same order or magnitude as the coupled signal having the transmitted polarization for certain slot orientations.

Should a higher order solution for the mutual coupling parameters be required, some additional evidence uncovered in this study will help in simplifying the analysis by avoiding the need of solving the full-vector diffraction probelm. Instead of computing the four-port scattering matrix, $S_{13}$ was computed assuming that only the two ports (1) and (3) were coupled, and then $S_{14}$ 


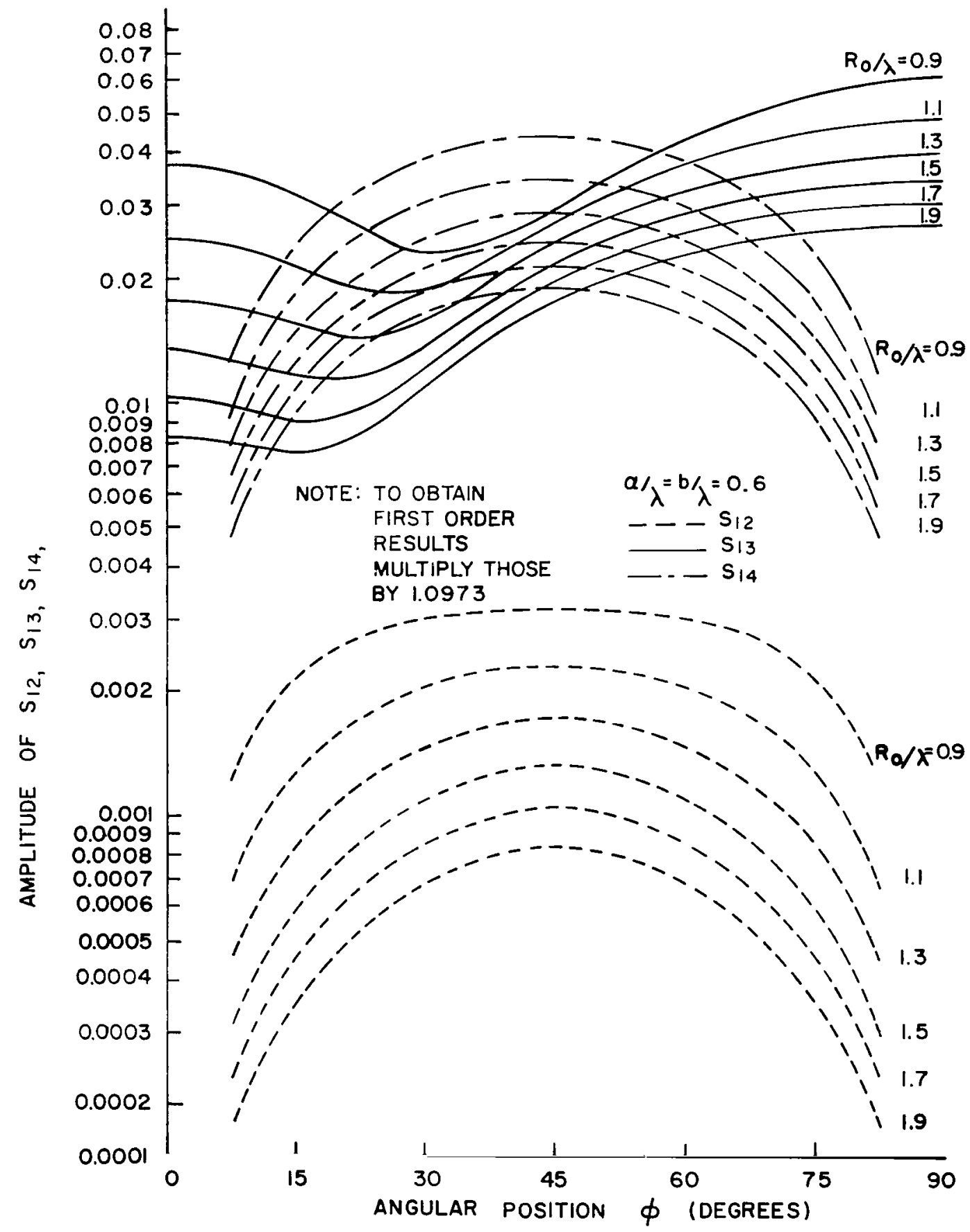

Figure 5A.- Amplitude and phase of the waveguide scattering parameters using improved first-order theory, amplitude of $\mathrm{S}_{12}, \mathrm{~S}_{13}, \mathrm{~S}_{14}$ 


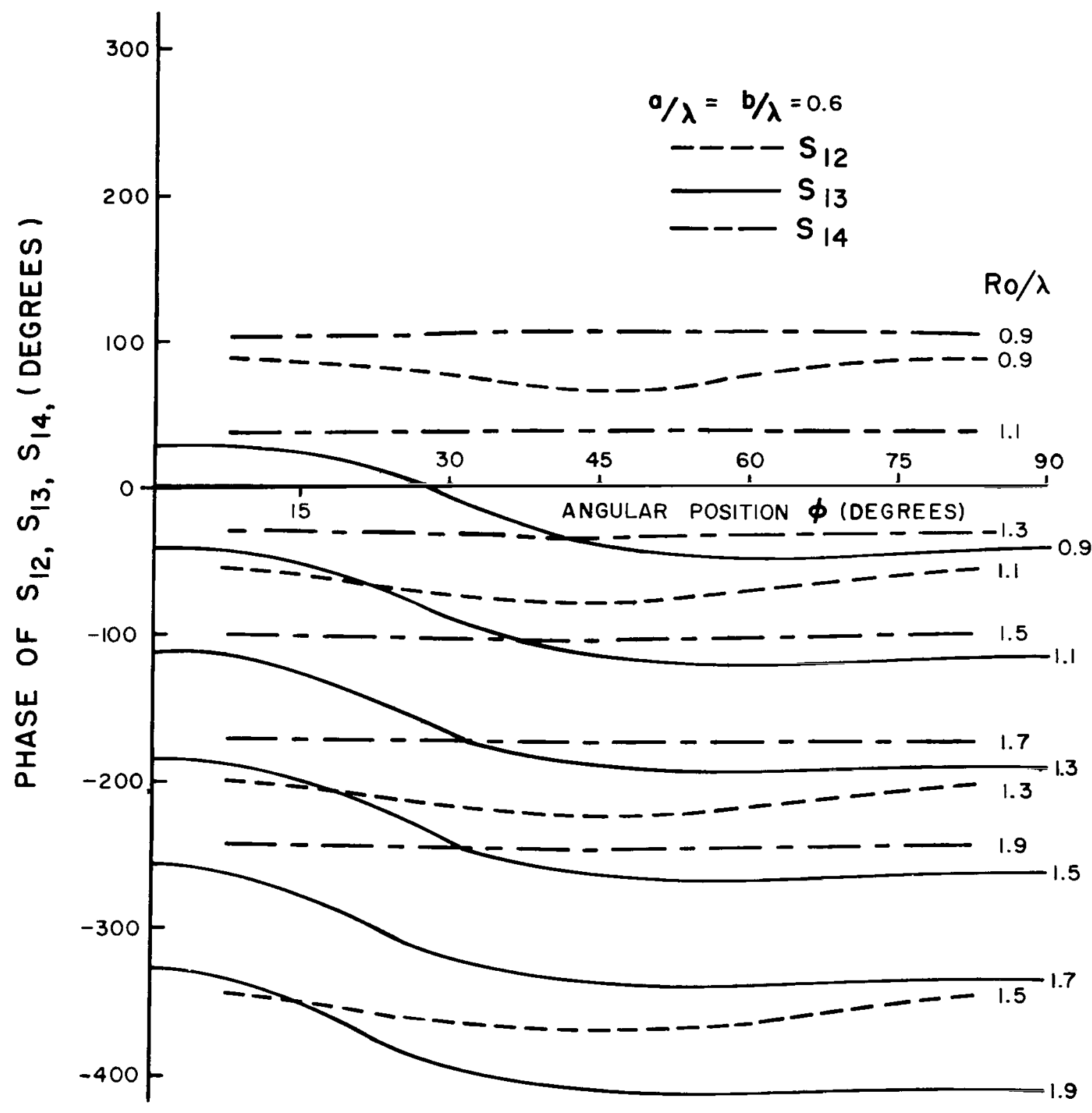

Figure 5B.- Amplitude and phase of the waveguide scattering parameters using improved first-order theory, phase of $\mathrm{S}_{12}, \mathrm{~S}_{13}, \mathrm{~S}_{14}$ 
is computed assuming that only ports (1) and (4) were coupled. The coupling between ports (2) and (3) or (4) was computed in the same manner using the following equation derived from Eq. for these approximate cases:

$$
\left.s_{m n}\right|_{m \neq n} \doteqdot \frac{-2 y_{m n}}{\left(1+y_{m m}\right)\left(1+y_{n n}\right)-y_{m n}^{2}}
$$

This approximation cannot be used to compute the secondorder cross-polarized terms $\mathrm{S}_{12}$ and $\mathrm{S}_{34}$ because $\mathrm{y}_{12}$ and $\mathrm{y}_{34}$ are zero. The other coupling parameters computed in this manner differ so little from those computed using the full four-port amittance matrix that they could not be distinguished in the accompanying curves. The same two-port model can be used to compute the reflection coefficients $S_{\mathrm{mm}}$ presented at each junction, but here it must be remembered that each of the two-port calculations contains the self-admittance term and so one must subtract

$$
\mathrm{s}_{\mathrm{mm}_{\text {I solated }}} \mid=\frac{\left(1-\mathrm{y}_{\mathrm{mm}}\right)}{\left(1+\mathrm{y}_{\mathrm{mm}}\right)}
$$

from the sum of the two $s_{m m}$ calculations computed using the two-port networks.

These approximate results are valid because the second-order orthogonal mode coupling coefficients $\left(S_{12}\right.$ and $\left.S_{34}\right)$ are very small. Since they are not a result of the coupling model used here, it seems likely that a similar approximation can be used in the derivation of coupling formulas if higher order mode solutions are desired.

Concerning the need for higher order approximations, the data presented in this paper show that the formulation used here should suffice to compute the parameters of most phased arrays. Whether it will suffice to describe the anomalous behavior which has been recently reported must be determined by further study.

Electronics Research Center

National Aeronautics and Space Administration

Cambridge, Massachusetts, December 1968

125-21-02-49 


\section{REFERENCES}

1. Mailloux, R. J.: Radiation and Near Field Coupling between Two Collinear Open-Ended Waveguides. IEEE Transactions on Antennas and Propagation, vol. AP-17, No. I, January 1969.

2. Mailloux, R. J.: Radiation and Coupling between Two Collinear Open-Ended Waveguides. NASA Technical Note TN D-4656, July 1968 .

3. Farrel1, G. F., Jr., and Kuhn, D. H.: Mutual Coupling Effects of Trianqular-Grid Arrays by Modal Analysis. IEEE Transactions on Antennas and Propagation, vol. AP-14, September 1966, pp. 652-654.

4. Farrell, G. F., Jr., and Kuhn, D. H.: Mutual Coupling in Infinite Arrays of Rectangular Waveguide Horns. IEEE Transactions on Antennas and Propagation, vol. AP-16, No. 4, July 1968, pp. 405-414.

5. Galindo, V., and Wu, C. P.: Properties of a Phased Array of Rectangular Waveguides with Thick Walls. IEEE Transactions on Antennas and Propagation, vol. AP-14, March 1966 , pp. 149-158.

6. Wu, C. P., and Galindo, V.: Properties of a Phased Array of Rectangular Waveguides with Thin Walls. IEEF Transactions on Antennas and Propagation, vol. AP-14, March 1966 , pp. 163-173.

7. Morse, P. M., and Feshbach, H.: Methods of Theoretical Physics. McGraw-Hill Book Co., New York, 1953, pp. 467 and 483 .

8. Albin, D. H., Nakatani, D. T., and Villeneuve, A. T.: On the Use of Experimental and Computational Techniques in Analyzing the Performance of Scanned Arrays. Scientific Report No. 9 on Contract AF 19 (628)-4349 (AFCRL-68-0297), March 1968.

9. Lewin, L.: Advanced Theory of Waveguides. Iliffe and Sons, Ltd., London, Chapter 6, 1951.

10. Levis, C.: Variational Calculations of the Impedance Parameters of Coupled Antennas. Report 667-16 (Contract AF 33 (616)-3353), Ohio State Research Foundation (AD-109370), 15 August 1956.

11. Amitay, N., Butzien, P. E., and Heidt, R. C.: Match Optimization of a Two-Port Phased Array Antenna Element. IEEE Transactions on Antennas and Propagation, vol. AP-16, No. 1, January 1968 , pp. 47-57. 
12. Marcuvitz, N.: Waveguide Handbook. MIT Radiation Lab. Series, vol. 10, McGraw-Hill Publishing Co., New York, 1950.

13. Lyon, J. A. M., Kalafus, R. M., Kwon, Y. K., Diegenis, C. J., Ibrahim, M. A. H., and Chen, C. C.: Derivation of Aerospace Antenna Coupling Factor Interference Prediction Techniques. Tech. Report AFAL-TR-66-57, University of Michigan, Radiation Laboratory, April 1966.

14. Harrington, R. F.: Field Computation by Moment Methods. The Macmillan Company, New York, 1968.

15. Mailloux, R. J., and LaRussa, F. J.: A Microwave Phase Bridge Technique for Measuring the Mutual Coupling of Identical Coupled Antennas. IEEE Transactions of Microwave Theory and Techniques, vol. MTT-16, February 1968. 


\section{APPENDIX I}

Throughout this paper, two orthogonal modes $\left(\mathrm{H}_{10}\right.$ and $\left.\mathrm{H}_{01}\right)$ are used to describe the fields in each waveguide. The field expansion used is that of Marcuvity (ref. 9, Ch. 1-2). The subscript notation is

Waveguide No. I: $E t_{1}=\sum_{i=1,2} v_{i}(z) \bar{e}_{i}\left(x_{1}, y_{1}\right)$;

$$
H t_{1}=\sum_{i=1,2} I_{i}(z) \bar{h}_{i}\left(x_{1}, y_{1}\right)
$$

Waveguide No. 2: $\quad E t_{2}=\sum_{i=3,4} V_{i}(z) \bar{e}_{i}\left(x_{2}, y_{2}\right)$;

$$
H t_{2}=\sum_{i=3,4} I_{i}(z) \bar{h}_{i}\left(x_{2}, y_{2}\right)
$$

The $\mathrm{H}$ mode functions $\bar{e}_{i}$ are defined by:

$$
\bar{h}_{i}=\hat{z} \times e_{i} \text { and } \bar{e}_{i}=\hat{z} \times \nabla_{t} \Psi_{i}
$$

where

$$
\left\{\begin{array}{l}
\Psi_{1} \\
\Psi_{3}
\end{array}\right\}=-\frac{2 a}{\pi b}\left\{\begin{array}{l}
\sin \frac{\pi x_{1}}{a} \\
\sin \frac{\pi x_{2}}{a}
\end{array}\right\}
$$

and

$$
\left\{\begin{array}{l}
\Psi_{2} \\
\Psi_{4}
\end{array}\right\}=-\frac{2 b}{\pi a}\left\{\begin{array}{c}
\sin \frac{\pi y_{1}}{b} \\
\sin \frac{\pi y_{2}}{b}
\end{array}\right\}
$$


The fields in free space are expanded in terms of the freespace Dyadic Greens function $\Gamma^{\circ}$ using the following relations:

$$
\begin{aligned}
& \bar{B}(\bar{r})=j 2 \omega \mu \varepsilon \sum_{p=1,2} \int_{S_{p}} \Gamma^{O}\left(\bar{r}, \bar{r}^{\prime}\right) \cdot(\hat{z} \times \bar{E}) d S_{p}^{\prime} \\
& \bar{E}(\bar{r})=2 \sum_{p=1,2} \int_{S_{p}} \bar{\nabla} G\left(\bar{r}, \bar{r}^{\prime}\right) \times(\hat{z} \times E) d S_{p}^{\prime}
\end{aligned}
$$

where

$$
\underline{\Gamma}^{O}\left(\bar{r}, \bar{r}^{\prime}\right)=\left(\underline{U}+\frac{I}{k_{0}^{2}} \underline{\nabla \nabla}\right) G\left(\bar{r}, \bar{r}^{\prime}\right)
$$

and

$$
\begin{aligned}
& G\left(r, r^{\prime}\right)=\frac{e^{-j k_{0}\left|\bar{r}-\bar{r}^{\prime}\right|}}{4 \pi\left|\bar{r}-\bar{r}^{\prime}\right|} \\
& \left|\bar{r}-\bar{r}^{\prime}\right|=\sqrt{\left(x-x^{\prime}\right)^{2}+\left(y-y^{\prime}\right)^{2}+z^{2}}
\end{aligned}
$$

The admittance dyad is defined below:

$$
\underline{\underline{y}}\left(\bar{r}, \bar{r}^{\prime}\right)=j 2 \omega \varepsilon \underline{\Gamma}^{\circ}\left(\bar{r}, \bar{r}^{\prime}\right)
$$


The constants $c_{1}$ and $c_{2}$ are dimensionless and defined below. The characteristic admittances for the various model waveguides are also defined below.

$$
\begin{aligned}
& c_{1}=\frac{j}{2 \pi^{2}} \frac{1}{(a / \lambda)(b / \lambda)} \frac{1}{\sqrt{1-\left[\frac{\lambda}{2 a}\right]^{2}}} \\
& c_{2}=\frac{j}{2 \pi^{2}} \frac{1}{(a / \lambda)(b / \lambda)} \frac{1}{\sqrt{1-\left[\frac{\lambda}{2 b}\right]^{2}}} \\
& y_{1}=y_{3}=\sqrt{\frac{\varepsilon_{0}}{\mu_{0}}} \sqrt{1-\left[\frac{\lambda}{2 a}\right]^{2}} \\
& y_{2}=y_{4}=\sqrt{\frac{\varepsilon_{0}}{\mu_{0}}} \sqrt{1-\left[\frac{\lambda}{2 b}\right]^{2}}
\end{aligned}
$$

The parameters $\Psi_{A}$ and $\Psi_{B}$ are also dimensionless:

$$
\begin{aligned}
\Psi_{A}\left(D_{x^{\prime}}, D_{y^{\prime}}, \mathrm{b}\right) & =\int_{-a / 2 \lambda}^{a / 2 \lambda} \mathrm{dx} \int_{-b / 2 \lambda}^{b / 2 \lambda} d y \int_{-a / 2 \lambda}^{a / 2 \lambda} d x^{\prime} \int_{-b / 2 \lambda}^{b / 2 \lambda} d y^{\prime} \\
& \cos \frac{\pi x}{a} \cos \frac{\pi x^{\prime}}{a}\left(\frac{\partial^{2}}{\partial x^{2}}+4 \pi^{2}\right) G \\
\Psi_{B}\left(D_{x^{\prime}} a_{;} D_{y^{\prime}} b\right)= & \int_{-a / 2 \lambda}^{a / 2 \lambda} d x \int_{-b / 2 \lambda}^{b / 2 \lambda} d y \int_{-a / 2 \lambda}^{a / 2 \lambda} d x^{\prime} \int_{-b / 2 \lambda}^{b / 2 \lambda} d y^{\prime} \\
& \cos \frac{\pi y^{\prime}}{b} \cos \frac{\pi x}{a} \frac{\partial^{2}}{\partial x \partial y} G
\end{aligned}
$$


where

$$
G=G\left(\frac{D}{\lambda}, x^{\prime}-x ; \frac{D}{\lambda}, y^{\prime}-y\right)=\frac{e^{-j 2 \pi r} \lambda}{r_{\lambda}}
$$

and

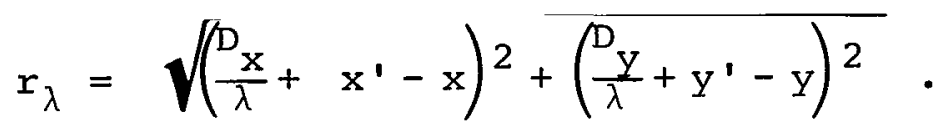

These basic integrals may be simplified somewhat by integrating them by parts and using trigonometric identities. After considerable manipulation, they may be written in the forms shown below:

$$
\left\{\begin{array}{l}
{ }^{\Psi_{A}} \\
\Psi_{B}
\end{array}\right\}=\int_{\tau=-a / 2 \lambda}^{\mathrm{a} / 2 \lambda} \mathrm{d \tau} \int_{\sigma=-b / 2 \lambda}^{\mathrm{b} / 2 \lambda} \mathrm{d \sigma}\left\{\begin{array}{l}
\mathrm{f}_{\mathrm{A}} \\
\mathrm{f}_{\mathrm{B}}
\end{array}\right\}
$$

where

$$
\begin{aligned}
\mathrm{f}_{\mathrm{A}}(\tau, \sigma)= & \frac{\pi^{2}}{2}[-\mathrm{u}(\tau-\mathrm{a} / 2 \lambda)-\mathrm{v}](\sigma-\mathrm{b} / 2 \lambda) \mathrm{G}_{\mathrm{A}} \\
& +\frac{\pi^{2}}{2}[u(\tau-a / 2 \lambda)+v](\sigma+b / 2 \lambda) \mathrm{G}_{\mathrm{B}} \\
& +\frac{\pi^{2}}{2}[-u(\tau+a / 2 \lambda)-v](\sigma-b / 2 \lambda) \mathrm{G}_{C} \\
& +\frac{\pi^{2}}{2}[u(\tau+a / 2 \lambda)+v](\sigma+b / 2 \lambda) \mathrm{G}_{D}
\end{aligned}
$$

and

$$
f_{B}(\tau, \sigma)=\cos \frac{\pi \tau}{a / \lambda} \cos \frac{\pi \sigma}{b / \lambda}\left[G_{A}-G_{B}-G_{C}+G_{D}\right]
$$


Other parameters are defined below:

$$
\begin{aligned}
G_{A} & =G\left(D_{x / \lambda}, \tau+a / 2 \lambda ; D_{y} / \lambda, \sigma+b / 2 \lambda\right) \\
G_{B} & =G\left(D_{x / \lambda}, \tau+a / 2 \lambda ; D_{y / \lambda}, \sigma-b / 2 \lambda\right) \\
G_{C} & =G\left(D_{x / \lambda}, \tau-a / 2 \lambda ; D_{y / \lambda}, \sigma+b / 2 \lambda\right) \\
G_{D} & =G\left(D_{x / \lambda}, \tau-a / 2 \lambda ; D_{y / \lambda}, \sigma-b / 2 \lambda\right) \\
u & =\left[4-(\lambda / a)^{2}\right] \sin \frac{\pi \tau}{a / \lambda} \\
v & =\left[4+(\lambda / a)^{2}\right] \frac{a}{\pi \lambda} \cos \frac{\pi \tau}{a / \lambda}
\end{aligned}
$$




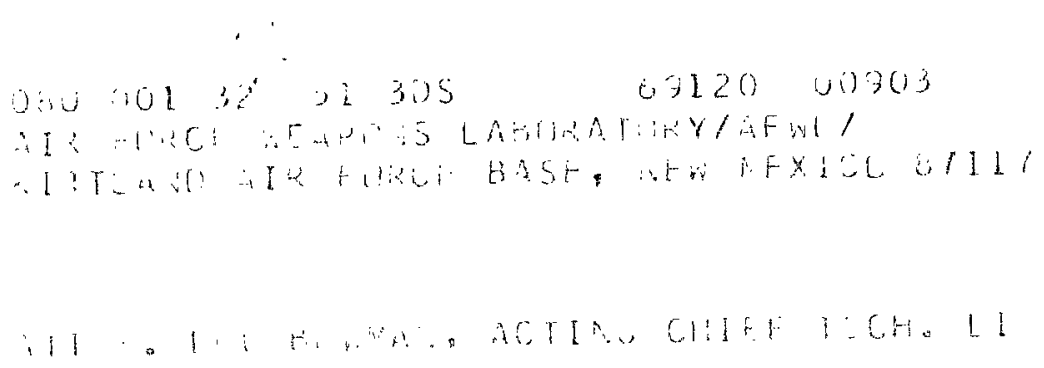

"The aeronautical and space activities of the United States shall be conducted so as to contribute. . . to the expansion of buman knowledge of phenomena in the atmosphere and space. The Administration shall provide for the widest practicable and appropriate dissemination of information concerning its activities and the results thereof."

\section{NASA SCIENTIFIC AND TECHNICAL PUBLICATIONS}

TECHNICAL REPORTS: Scientific and technical information considered important, complete, and a lasting contribution to existing knowledge.

TECHNICAL NOTES: Information less broad in scope but nevertheless of importance as a contribution to existing knowledge.

TECHNICAL MEMORANDUMS:

Information receiving limited distribution because of preliminary data, security classification, or other reasons.

CONTRACTOR REPORTS: Scientific and technical information generated under a NASA contract or grant and considered an important contribution to existing knowledge.
TECHNICAL TRANSLATIONS: Information published in a foreign language considered to merit NASA distribution in English.

SPECIAL PUBLICATIONS: Information derived from or of value to NASA activities. Publications include conference proceedings, monographs, data compilations, handbooks, sourcebooks, and special bibliographies.

\section{TECHNOLOGY UTILIZATION}

PUBLICATIONS: Information on technology used by NASA that may be of particular interest in commercial and other non-aerospace applications. Publications include Tech Briefs, Technology Utilization Reports and Notes, and Technology Surveys.

Details on the availability of these publications may be obtained from:

SCIENTIFIC AND TECHNICAL INFORMATION DIVISION

NATIONAL AERONAUTICS AND SPACE ADMINISTRATION

Washington, D.C. 20546 